\title{
An evaluation of a bioelectrical impedance analyser for the estimation of body fat content
}

\author{
R. J. Maughan PhD \\ Department of Environmental and Occupational Medicine, University Medical School, Foresterhill, Aberdeen, \\ UK
}

\begin{abstract}
Measurement of body composition is an important part of any assessment of health or fitness. Hydrostatic weighing is generally accepted as the most reliable method for the measurement of body fat content, but is inconvenient. Electrical impedance analysers have recently been proposed as an alternative to the measurement of skinfold thickness. Both these latter methods are convenient, but give values based on estimates obtained from population studies. This study compared values of body fat content obtained by hydrostatic weighing, skinfold thickness measurement and electrical impedance on 50 (28 women, 22 men) healthy volunteers. Mean(s.e.m.) values obtained by the three methods were: hydrostatic weighing, 20.5(1.2)\%; skinfold thickness, $21.8(1.0) \%$; impedance, $20.8(0.9) \%$. The results indicate that the correlation between the skinfold method and hydrostatic weighing $(0.931)$ is somewhat higher than that between the impedance method and hydrostatic weighing $(0.830)$. This is, perhaps, not surprising given the fact that the impedance method is based on an estimate of total body water which is then used to calculate body fat content. The skinfold method gives an estimate of body density, and the assumptions involved in the conversion from body density to body fat content are the same for both methods.
\end{abstract}

Keywords: Body composition, bioelectrical impedance, hydrostatic weighing, anthropometry, skinfold thickness, body fat

There is a great deal of interest in the measurement of body fat content in view of the well-established association between an increased degree of fatness and a variety of disease processes. Direct measurements of fat content are not possible except after death, and in humans only a limited number of such measurements have been made. A variety of indirect methods has therefore been proposed to allow estimates of body fat content to be made. Hydrostatic weighing, which measures body density and assumes that the body consists of fat and lean compartments, each with a specific and constant density, is generally recognized as the most 'accurate' method, but this may be misleading as none of these indirect methods has ever been validated directly ${ }^{1}$.

Address for correspondence: R. J. Maughan PhD, Department of Environmental and Occupational Medicine, University Medical School, Foresterhill, Aberdeen AB9 2ZD, UK

(C) 1993 Butterworth-Heinemann Ltd 0306-3674/93/010063-04
Many other methods for the assessment of body composition are available. Measurement of total body water, based on isotope or solute dilution methods, allows an estimate of fat content, assuming that the fat component is water-free and further assuming a constant water content of the lean component. Based on this inverse relationship between water content and fat content of the body, a variety of methods which estimate body water content can then be employed to estimate body fat content. These methods include total body potassium measured from potassium- 40 counting, and the more recently introduced measurement of bioelectrical impedance analysis (BIA).

Several studies have compared two or more of these different methods ${ }^{2-4}$. As expected, there are strong correlations between the results obtained by the different methods when populations are examined, but there are also discrepancies between the results obtained by different methods for some individuals. Again this is not surprising in view of the different assumptions involved when different methods are applied. The inconvenience of the hydrostatic weighing method, however, together with the requirement for a high degree of subject cooperation, restricts its use, and makes the use of alternative methods inevitable where large numbers are to be studied.

The aim of the present study was to compare results obtained by BIA and skinfold thickness measurement with those obtained by hydrostatic weighing in a population of healthy adult subjects.

\section{Methods}

A total of 50 volunteers was recruited to act as subjects: of these, 28 were women and 22 men. Descriptive data are given in Table 1. Subjects were volunteers recruited from laboratory staff and from a local aerobics class, which may account for the fact that body fat content is rather lower than might be expected for the normal population. All measurements were carried out by the same investigator.

Subjects reported to the laboratory without any restriction on prior food and fluid intake, but subjects were requested to avoid strenuous exercise in the few hours preceding the test. Impedance measurements are known to be sensitive to hydration status, and 
Table 1. Anthropometric characteristics of subjects

\begin{tabular}{lccc}
\hline & $\begin{array}{c}\text { All } \\
(\mathrm{n}=50)\end{array}$ & $\begin{array}{c}\text { Men } \\
(\mathrm{n}=22)\end{array}$ & $\begin{array}{c}\text { Women } \\
(\mathrm{n}=28)\end{array}$ \\
\hline Age (years) & $33(1)$ & $36(2)$ & $30(1)$ \\
Height $(\mathrm{m})$ & $1.69(0.01)$ & $1.76(0.01)$ & $1.63(0.01)$ \\
Weight $(\mathrm{kg})$ & $66.5(1.5)$ & $76.1(1.4)$ & $59.0(1.3)$ \\
\hline
\end{tabular}

Values are mean(s.e.m.)

exercise-induced dehydration is a potential confounding factor. All three measurements were made in sequence within a period of $1 \mathrm{~h}$. Impedance was measured using a Bodystat 500 analyser (Bodystat, Douglas, Isle of Man) according to the manufacturer's instructions. Measurements were made with the subjects in a supine position and fully clothed except for removal of the right shoe and sock to allow placement of the electrodes. Care was taken to ensure that the legs were apart and the arms were not touching the sides of the body, in accordance with the manufacturer's instructions. Body fat content was calculated from the impedance measurement obtained using software release version 6.0; the manufacturers do not give details of the equation used. Whole body resistive index (WBRI) was calculated as $H t^{2} / R$, where $H t$ is the height in metres and $R$ is the impedance value in ohms. Duplicate measurements were made on a subgroup of eight subjects at an interval of 1-3 days. The mean difference between the repeated measurements was 0.6 (range -0.9 to +2.9 ) $\%$ body fat; the coefficient of variation for the paired measurements was $6.8 \%$.

Body weight was recorded with the subjects nude immediately before hydrostatic weighing and was read to the nearest $0.01 \mathrm{~kg}$. Height was recorded to the nearest $0.01 \mathrm{~m}$. Hydrostatic weighing was carried out as previously described ${ }^{4}$. Briefly, subjects were seated in a chair suspended in a tank of water maintained at a temperature of $34-36^{\circ} \mathrm{C}$. The chair was suspended from a steel plate to which were attached four strain gauges arranged to form a Wheatstone bridge circuit balanced with a $9 \mathrm{~V}$ d.c. supply. The amplified output of the circuit was displayed on a pen recorder. The system was calibrated immediately after use on each occasion using known weights. The subject sat immersed to the neck, and submerged by bending forward after a forced maximal expiration. After 3-4 measurements of underwater weight, residual volume was determined by an oxygen rebreathing method as described by Wilmore et al $l^{5}$. After two or three measurements of residual volume, a further two or three measurements of underwater weight were made. Body fat content, expressed as a percentage of body weight, was calculated using the formula of Siri ${ }^{6}$ :

Body fat $(\%)=((4.95 /$ density $)-4.5) \times 100$

Duplicate measurements were made on a subgroup of eight subjects at an interval of 1-3 days. The mean difference between the repeated measurements was 0.5 (range -1.4 to +0.3 ) $\%$ body fat; the coefficient of variation for the paired measurements was $2.3 \%$.
Skinfold thickness was measured using Harpenden skinfold calipers at the sites described by Durnin and $\operatorname{Rahaman}^{7}$; these are biceps, triceps, subscapula and suprailiac. Measurements were made in triplicate and the tables of Durnin and Rahaman were used to calculate body fat content. Duplicate measurements were made on a subgroup of eight subjects at an interval of 1-3 days. The mean difference between the repeated measurements was 0.2 (range -1.3 to $+0.6) \%$ body fat; the coefficient of variation for the paired measurements was $2.6 \%$.

\section{Data analysis}

Group data are presented as mean(s.e.m.) unless otherwise indicated. Results obtained by the three methods were compared by analysis of variance. The results obtained by skinfold thickness were compared with those obtained by hydrostatic weighing by linear regression analysis using a commercially available statistical programme (Minitab, State College, Pennsylvania, USA). The BIA method estimates body fat by estimation of body water and the calculation of body fat involves a different set of assumptions from those based on estimates of body density. It is therefore inappropriate to assume that the values obtained from hydrostatic weighing are necessarily correct, and the agreement between the results obtained by these two methods was asssessed as described by Bland and Altman ${ }^{8}$. This involves examination of the relationship between the differences between the two methods and the average of the values obtained by the two methods.

\section{Results}

Estimates of body fat content made by the three methods are presented in Table 2. Analysis of variance indicated that there was no difference between the three methods. Linear regression analysis showed good agreement between the three methods when all 50 subjects were included in the analysis; the correlation coefficient between hydrostatic weighing and skinfold thickness was 0.931, between hydrostatic weighing and bioelectric impedance 0.830 , and between skinfold thickness and bioelectric impedance 0.842 . When the sexes were compared separately, the agreement between hydro-

Table 2. Body fat content, expressed as a percentage of body weight, estimated by hydrostatic weighing $(\mathrm{HW})$, skinfold thickness (SFT) and bioelectric impedance (BIA)

\begin{tabular}{lccc}
\hline & $\begin{array}{c}\text { AlI } \\
(\mathrm{n}=50)\end{array}$ & $\begin{array}{c}\text { Men } \\
(\mathrm{n}=22)\end{array}$ & $\begin{array}{c}\text { Women } \\
(\mathrm{n}=28)\end{array}$ \\
\hline HW & $20.5(1.2)$ & $13.4(1.3)$ & $26.2(0.9)$ \\
& $(2.0-38.0)$ & $(2.0-22.7)$ & $(18.1-38.0)$ \\
SFT & $21.8(1.0)$ & $15.1(0.7)$ & $27.0(0.8)$ \\
& $(8.1-37.5)$ & $(8.1-19.7)$ & $(17.3-37.5)$ \\
BIA & $20.8(0.9)$ & $15.5(0.9)$ & $25.0(0.8)$ \\
& $(9.0-33.0)$ & $(9.0-24.1)$ & $(15.1-33.0)$ \\
\hline
\end{tabular}

Values are mean(s.e.m.) and (range) 
static weighing and skinfold thickness remained good, 0.874 for the men and 0.822 for the women. The agreement between hydrostatic weighing and bioelectric impedance was less good, 0.528 for men and 0.650 for women.

WBRI was closely related to the fat-free mass (FFM) calculated from body weight and body fat content estimated from hydrostatic weighing ( $r=0.953$, Figure 1). Linear regression analysis gives the following relationship

$\mathrm{FFM}=0.81+(9419 \times \mathrm{WBRI})$

Inclusion of other variables in the regression analysis leads to an improvement in the relationship. Addi-

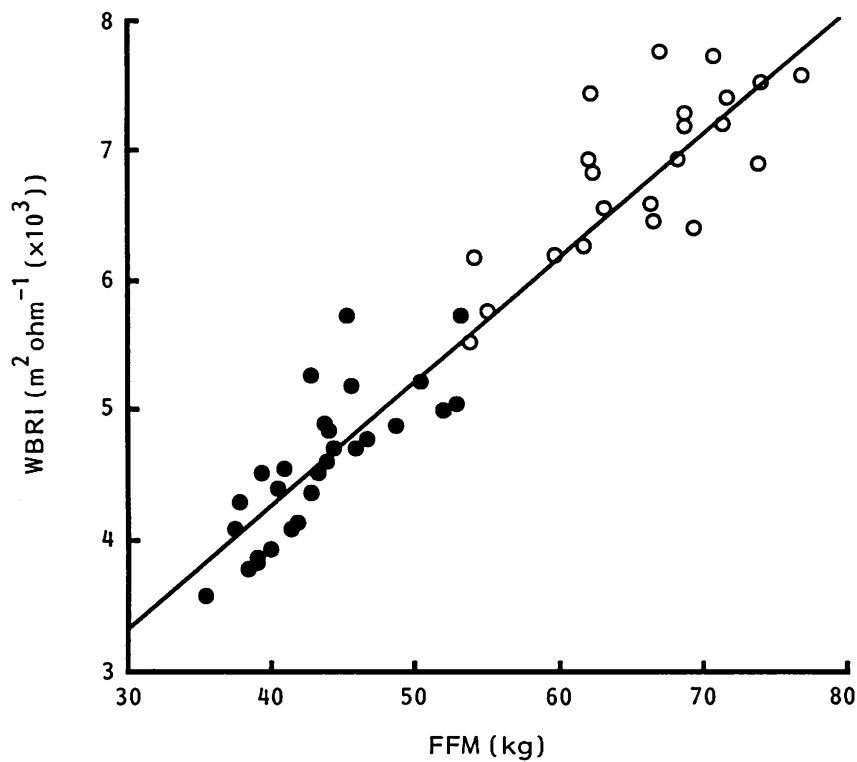

Figure 1. Relationship between whole body resistive index (WBRI), calculated as $H t^{2} / R$ where $H t$ is height in metres and $R$ is the impedance value in ohms, and the fat-free mass calculated from body weight and the body fat content estimated by hydrostatic weighing. 0 , women; $\mathrm{O}$, men

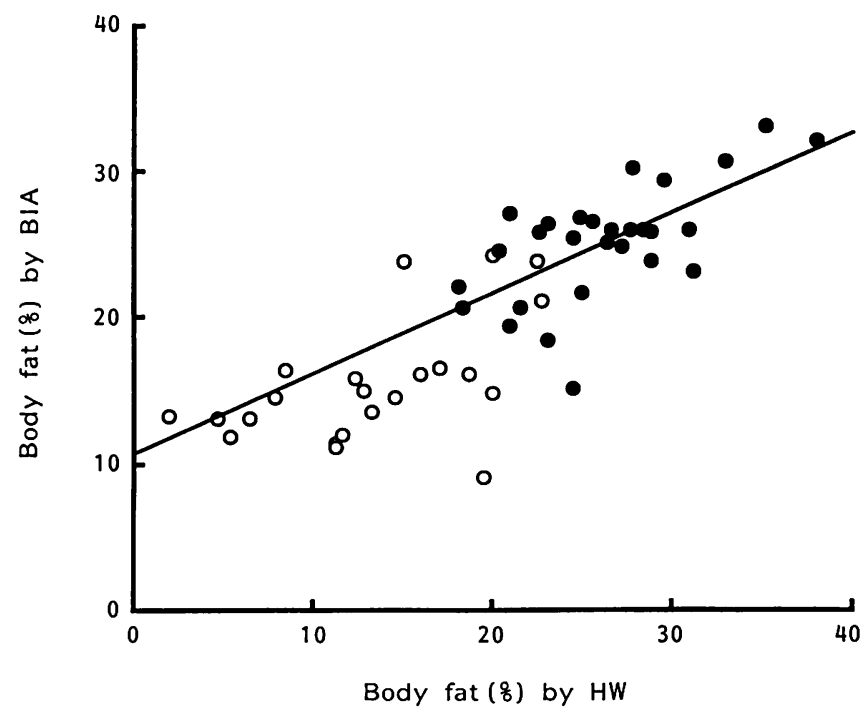

Figure 2. Relationship between body fat content as a percentage of body weight determined by bioelectric impedance analysis (BIA) and by hydrostatic weighing $(\mathrm{HW})$. $\mathrm{O}$, women; $\bigcirc$, men tion of sex (with a dummy code: $1=$ male, $2=$ female), age (years), height $\left(\mathrm{m}^{2}\right)$, weight $(\mathrm{kg})$ and impedance (ohms) gives the following relationship, where $R^{2}=95.1 \%$ :

$\mathrm{FFM}=22.5-(8.84 \times$ sex $)-(0.744 \times$ age $)$

$+(0.289 \times$ weight $)-(0.0222 \times$ impedance $)+\left(13.9 \times \mathrm{Ht}^{2}\right)$

Comparison of the values for percentage body fat content obtained by hydrostatic weighing and bioelectric impedance was carried out using the procedures recommended by Bland and Altman ${ }^{8}$ and the results for the whole subject group are shown in Figure 2. The difference between the values obtained by the two methods was linearly correlated with the mean of the values obtained by the two methods ( $r=$ $0.457, P<0.001)$. For individuals with a low body fat content, the impedance method gives a higher value than that obtained by hydrostatic weighing, but the situation is reversed when body fat content is high. Comparing the sexes separately using this analysis indicates a similar trend for the men $(r=0.383, P<$ $0.1)$ but not for the women $(r=0.231)$.

Similar results are obtained when the values for FFM obtained by the two methods are compared. Linear regression analysis suggests a close relationship between FFM calculated by the Bodystat software and FFM calculated from the hydrostatic weighing data $(r=0.968)$. However, there is a tendency $(r=0.329, P<0.05)$ for the FFM determined by impedance to be lower than the mean of the values estimated by the two methods for subjects with low FFM and for the value estimated by impedance to be higher than the mean value when FFM is high.

\section{Discussion}

Because of the convenience of the method, the use of bioelectric impedance measurements for the estimation of body fat content in man is now widespread. A large number of studies has compared results obtained using this technique with those obtained by other methods. These comparisons fall into two categories: first, those in which impedance is used alone or together with other variables to produce the best possible predictive equation, and second, those which use published predictive equations against which comparisons are made.

When impedance measurements are compared with estimates of total body water obtained by dilution methods, the correlations between the two methods are generally good. Lukaski ${ }^{9}$ reported a correlation of 0.97 between impedance and total body water determined by deuterium dilution in a group of 33 men and women; the predictive equation developed included body weight, height and age. The equations derived by Kushner and Schoeller ${ }^{10}$ included weight, height and sex, and also found a close relationship $(r=0.99)$ when comparing these variables in a group of 20 obese and 20 non-obese individuals. Others, however, have obtained relationships that are less good - Van Loan and Mayclin ${ }^{11}$ included height, weight, sex and age in a predictive equation derived from 188 men and women and found a correlation coefficient of 0.87 . 
Most evaluations of the impedance method have compared it with the fat free body mass (FFM). Again, impedance has been used in combination with a variety of other anthropometric variables to produce predictive equations. These studies have recently been reviewed by Van Loan ${ }^{12}$. The correlations reported between WBRI and FFM are generally similar to the value of 0.953 obtained in the present study.

There appear to have been fewer studies comparing estimates of body fat content using bioelectrical impedance, together with the equipment manufacturer's predictive equations, with estimates of body fat obtained by other methods. There are difficulties in establishing the validity of the equations used by equipment manufacturers, as these are not normally made available to users. In the present study, however, the correlation between WBRI and FFM estimated by hydrostatic weighing $(r=0.953)$ was better than that between percentage body fat derived from hydrostatic weighing and that estimated by the impedance method $(r=0.830)$. This suggests that the population from which the predictive equation used by the manufacturers was derived may differ from the present population.

In the present study, the skinfold method appears to agree more closely with the hydrostatic weighing method than does the BIA method. There may be several factors responsible for this. First, the hydrostatic weighing method estimates body density, and fat content is derived by assuming constant and standard densities of fat and of the fat-free mass. There are undoubtedly errors in this assumption ${ }^{1,13}$, and it may be unreasonable to compare other methods against hydrostatic weighing. An example of these errors is apparent in the values obtained by hydrostatic weighing for some subjects in the present study: three subjects, all of whom were professional soccer players, obtained values of $2.0 \%, 4.7 \%$ and $6.5 \%$ for body fat content. The same three subjects recorded values of $13.3 \%, 13.2 \%$ and $13.2 \%$ respectively by impedance measurement and $11.5 \%, 10.9 \%$ and $14.0 \%$ respectively by skinfold thickness measurement. It seems certain that the hydrostatic weighing method underestimated body fat content in these subjects. The reason for this may be an increased bone and muscle mass, leading to an increased lean tissue density, in these individuals as a result of the stresses associated with training for competitive soccer. The use of a single value for the density of the fat-free mass in all individuals is potentially the source of major errors in the use of the hydrostatic weighing technique ${ }^{14}$.

The impedance method estimates fat-free mass from an estimate of body water content, and the assumptions involved in calculating fat content from total body water will inevitably lead to differences between these methods, even if total body water is measured correctly ${ }^{4}$. It appears that many of the equations used for the prediction of body fat content from impedance measurements may have been derived from comparisons against hydrostatic weighing without taking account of this fact. The skinfold thickness equations of Durnin and Rahaman ${ }^{7}$ which were used in this study were derived from a comparison between skinfold thickness and body density as determined by hydrostatic weighing, and might thus be expected to agree more closely.

The results appear to indicate that either skinfold thickness or bioelectrical impedance can be substituted for hydrostatic weighing where there is a need to determine body fat content. On this population of subjects, there was a slightly better agreement between results obtained by skinfold thickness and those from hydrostatic weighing than between impedance and hydrostatic weighing. This may be due to errors in the estimation of body fat by hydrostatic weighing rather than errors in the impedance method. The reproducibility of the impedance method was less good than that of the other two methods, but this probably reflects the relative inexperience of the operator with this technique.

\section{References}

1 Martin AD, Drinkwater DT. Variability in the measures of body fat; assumptions or technique? Sports Med 1991; 11: 277-88.

2 Womersley J, Boddy K, King PL, Durnin JVGA. A comparison of the fat-free mass of young adults estimated by anthropometry, body density and total body potassium content. Clin Sci 1972; 43: 469-75.

3 Kryzwicki HJ, Ward GM, Rahman DP, Nelson RA, Consolazio CF. A comparison of methods of estimating human body composition. Am J Clin Nutr 1974; 27: 1380-5.

4 McNeill GM, Fowler PA, Maughan RJ et al. Body fat in lean and overweight women estimated by six methods. $\mathrm{Br} J \mathrm{Nutr}$ 1991; 65: 95-103.

5 Wilmore JH, Vodak PA, Parr RB, Girandola RN, Billing JE. Further simplification of a method for determination of residual lung volume. Med Sci Sports Exerc 1980; 12: 216-18.

6 Siri WE. Body composition from fluid space and density: analysis of methods. In: Brozek J, Henschel A, eds. Techniques for Measuring Body Composition. Washington DC, USA National Academy of Science/National Research Council, $1961 ; 223-45$.

7 Durnin JVGA, Rahaman MM. The assessment of fat in the human body from measurements of skinfold thickness. $\mathrm{Br} J$ Nutr 1967; 21: 681-9.

8 Bland JM, Altman DG. Statistical methods for assessing agreement between two methods of clinical measurement. Lancet 1986; i: 307-10.

9 Lukaski HC. Methods for the assessment of body composition: traditional and new. Am J Clin Nutr 1987; 46: 537-56.

10 Kushner RF, Schoeller DA. Estimation of total body water by bioelectrical impedance analysis. Am J Clin Nutr 1986; 44: 417-24.

11 Van Loan M, Mayclin P. Bioelectrical impedance analysis: is it a reliable estimator of lean body mass and total body water? Hum Biol 1987; 59: 299-309.

12 Van Loan M. Bioelectrical impedance analysis to determine fat-free mass, total body water and body fat. Sports Med 1990; 10: 205-17.

13 Lohman TG. Skinfolds and body density and their relation to body fatness: a review. Hum Biol 1981; 53: 181-225.

14 Martin AD, Drinkwater DT. Variability in the measures of body fat. Sports Med 1991; 11: 277-88. 